\title{
Entrelacs
}

ENTRELACS Cinéma et audiovisuel

$10 \mid 2013$

Le Toucher

\section{Le toucher dans le cinéma français des sensations}

\section{Sophie Walon}

\section{(2) OpenEdition}

Journals

Édition électronique

URL : https://journals.openedition.org/entrelacs/530

DOI : $10.4000 /$ entrelacs.530

ISSN : 2261-5482

Éditeur

Éditions Téraèdre

Référence électronique

Sophie Walon, «Le toucher dans le cinéma français des sensations », Entrelacs [En ligne], 10 | 2013,

mis en ligne le 12 septembre 2013, consulté le 21 septembre 2021. URL : http://

journals.openedition.org/entrelacs/530; DOI : https://doi.org/10.4000/entrelacs.530

Ce document a été généré automatiquement le 21 septembre 2021.

Tous droits réservés 


\title{
Le toucher dans le cinéma français des sensations
}

\author{
Sophie Walon
}

1 "Cinéma des sensations ", c'est ainsi que la critique a désigné un nouveau mouvement du cinéma français apparu dans les années quatre-vingt-dix lequel place le corps et ses sensations au cœur de son propos et de son esthétique. Cette nouvelle tendance cinématographique regroupe, entre autres, les films de Claire Denis, Bruno Dumont, Catherine Breillat, Philippe Grandrieux, Gaspar Noé, Bertrand Bonello, etc. Naturellement, puisqu'il s'intéresse aux corps et aux sensations, ce cinéma accorde une place privilégiée à l'exploration d'un large éventail d'expériences tactiles, des plus sensuelles aux plus aliénantes.

2 Ce débordement de sensations a parfois été taxé de pur formalisme voire de tactique sensationnaliste dans la lignée d'un article acerbe de James Quandt ${ }^{1}$ dans lequel le critique repère et dénonce avec véhémence l'émergence d'une nouvelle tendance du cinéma français, violente et obscène, qu'il propose d'appeler New French Extremity. Ce cinéma succombe selon lui à une logique du choc, à la provocation facile, gratuite et donc creuse. Il condamne ainsi ce cinéma qui semble déterminé à « remplir chaque plan de chair nubile ou perverse et à la soumettre à toutes sortes de pénétration, de mutilation et de profanation $»^{2}$.

3 Ces expériences limites du toucher (pénétration, mutilation, profanation) ne sont-elles effectivement que des stratégies commerciales destinées à appâter les spectateurs avides de sensations extrêmes ? Il est permis d'en douter car ces réalisateurs témoignent d'une certaine indépendance en terme de production et leurs films, de fait, n'attirent pas les masses, dépassant rarement la barre des 100000 spectateurs.

4 Dans son ouvrage sur le cinéma français des sensations (2007), Martine Beugnet a pris le contre-pied des analyses de Quandt en soulignant que ces réalisateurs ont su inventer une nouvelle voie pour le cinéma qui, évitant à la fois les écueils de l'abstraction pure et ceux des productions commerciales, ranime l'impact sensoriel et la nature transgressive du médium cinématographique. Martine Beugnet célèbre donc ce cinéma qui explore de 
manière originale « la capacité unique du cinéma à nous toucher à la fois viscéralement et intellectuellement $»^{3}$.

5 Ce type d'analyse reste rare, la littérature critique ou universitaire ayant tendance à ignorer l'impact viscéral du cinéma au profit d'explications rationalisantes ou psychologisantes. Cela peut peut-être s'expliquer par le fait qu'avant que le cinéma d'art ou d'auteur ne s'en empare comme c'est le cas avec le cinéma français des sensations, la représentation des corps et des sensations à l'écran ainsi que l'implication corporelle des spectateurs étaient le plus souvent associées à des genres cinématographiques tels que le film pornographique, le cinéma d'horreur ou le mélodrame. Or ces genres ont longtemps été considérés par les critiques et les universitaires comme des genres "bas », voire ignobles, indignes d'un intérêt théorique ${ }^{4}$.

6 Toutefois, ces interrogations sur la représentation des sensations au cinéma et la manière dont elles peuvent être signifiantes, jouissent d'un récent intérêt théorique dont témoignent les recherches de Vivian Sobchack, Laura U. Marks et Martine Beugnet sur lesquelles cet essai s'appuiera. Penser le toucher au cinéma permet donc de contribuer à ce déplacement de la théorie cinématographique. En effet, cette nouvelle approche du cinéma s'éloigne aussi bien des théories sémiotiques qui invitaient à lire le texte des films en se concentrant sur un déchiffrage rationalisant de leur structure narrative, que des théories psychanalytiques qui privilégiaient une compréhension symbolique de l'expérience cinématographique. S'intéresser à l'impact sensoriel des films, c'est-à-dire aux corps et aux sensations qu'ils représentent, à la matérialité des images et du son ainsi qu'aux réponses corporelles des spectateurs face au film, invite à réinterroger la nature de l'expérience cinématographique et la manière dont le sens d'un film émerge non seulement au niveau des signes et des symboles mais aussi dans la matière même des images et des sons, dans les sensations qui sont mises en scène et dans la façon dont elles touchent les spectateurs.

7 Dans cet essai, j'interrogerai donc les différents niveaux d'expériences tactiles qu'offre ce cinéma des sensations. J'examinerai d'abord les diverses modalités tactiles mises en scène à l'écran (le toucher des personnages), puis je soulignerai que ces films font de l'expérience cinématographique elle-même une expérience tactile à travers le régime haptique des images et du son (le toucher du film) et leur impact sensoriel (le toucher du spectateur).

\section{Le toucher des personnages : de la caresse à la déchirure, de la fusion à la destruction}

Ce cinéma des sensations, comme son nom le laisse supposer, s'intéresse au toucher et se concentre ainsi sur les expériences tactiles de ses personnages. Pour autant, cet accent porté sur le toucher, s'il n'a jamais été aussi radical, n'est pas nouveau : de Bresson à Duras, d'Hitchcock à Malick, de Bergman à Tarkovski, les expériences tactiles sont nombreuses et variées au cinéma. Mais dans le travail de ces réalisateurs, la narration ou du moins le régime discursif (qu'il soit spirituel, symbolique ou philosophique) restait souvent au cœur du projet cinématographique tandis que dans le cinéma des sensations, la polarité semble s'inverser. Les sensations, notamment tactiles, deviennent centrales; elles ne sont pas au service d'une histoire ou d'un discours, elles les constituent intrinsèquement. Ce déplacement est donc une affaire de degré et de focale. Dans le 
cinéma des sensations, l'expérience du toucher dans toutes ses formes passe littéralement au premier plan; les contacts et les organes du toucher (la main, la peau, le sexe) étant souvent filmés en gros plan et la question de la rencontre tactile interrogée de manière essentielle et non secondaire au sein de la narration.

En mettant en scène diverses expériences du toucher, ces films interrogent les êtres dans leur capacité d'ouverture au monde en filmant parfois les corps comme des interfaces poreuses, perméables qui permettent réellement d'aller à la rencontre de l'autre et parfois comme des limites infranchissables qui font obstacle à un échange véritable entre les êtres. En découlent les vécus variés du toucher que déclinent ces films, de l'épanouissement à la frustration jusqu'aux tentatives d'appropriation et de destruction de l'autre. De l'effleurement à l'étreinte, de la caresse au coup, du frôlement à la profanation, l'expérience du toucher est ainsi explorée dans ses modalités les plus fusionnelles comme les plus disjonctives.

10 Ces films ne présentent donc pas seulement des corps unis dans des expériences satisfaisantes, agréables et sensuelles, ce que la notion de toucher évoque spontanément. Nombreux sont ceux qui mettent en scène des expériences négatives, voire morbides, du toucher où le contact se vit sur le mode de la séparation et témoigne de la résistance ultime qu'opposent l'autre et le monde au désir d'union d'un sujet. Frapper, triturer, couper, mordre, enfoncer, percer, mutiler sont autant de modalités du toucher qui, loin d'inviter à la rencontre épanouissante ou à l'échange fusionnel, engendrent la dégradation voire la destruction de l'autre, suggerant un sentiment d'hostilité et de séparation irréductibles entre les êtres. Ces films balayent ainsi le large spectre des qualités du toucher et des affects qui peuvent y être associés, allant de la jouissance la plus grande aux souffrances les plus terribles. On soulignera de quelles intentions et significations ces expériences tactiles peuvent être solidaires ainsi que les figures de l'altérité qu'elles dessinent.

11 Naturellement, la mise en scène du toucher est souvent synonyme d'une exploration sensuelle des corps et des rapports humains. À cet égard, Lady Chatterley (2006) de Pascale Ferran constitue sans doute l'exemple le plus éloquent. À travers l'histoire de l'éveil sensuel de son personnage éponyme, le film est une ode au toucher, un éloge de la caresse. Adapté de l'œuvre de D. H. Lawrence, le film dévoile la découverte du désir et du plaisir par Constance, une jeune femme de la haute société dans l'Angleterre des années vingt. Alors qu'elle coule des jours monotones, enfermée dans son château et dans son mariage avec un époux infirme, elle fait la connaissance de Parkin, le garde-chasse du domaine, dont elle tombe éperdument amoureuse. Quand Constance, qui doit porter un ordre à Parkin, le surprend torse nu en train de faire sa toilette à l'air libre, elle est troublée par ce corps robuste, vigoureux qui contraste fortement avec celui, chétif, de son mari.

12 Le printemps suivant, partie cueillir des jonquilles, elle s'arrête à la cabane de Parkin pour se reposer. Dans cette séquence, les premiers mouvements du désir semblent inspirés par la nature, par la sérénité et la disponibilité sensorielles auxquelles elle invite. Le frémissement des feuilles, le parfum des fleurs, le flot de la rivière semblent réveiller une sensualité jusque-là endormie. D'ailleurs, quelques scènes plus tard, alors qu'elle court à la cabane, Constance s'arrête un instant contre un arbre pour reprendre son souffle et touche avec volupté la mousse épaisse qui le recouvre : le désir charnel que lui inspire Parkin semble d'abord trouver des substituts dans un contact de plus en plus sensuel avec le monde végétal. Constance multipliant ses visites à Parkin, un processus 
d'apprivoisement mutuel se développe alors entre cette femme maladroite et cet « ours mal léché ». En l'aidant dans son travail, elle entre, comme lui, en contact étroit avec la nature : elle boit désormais à même la source, travaille la terre, s'occupe des fleurs, caresse un poussin, etc. Un jour, elle enfile un gant de Parkin : bien que rêche à l'extérieur, on imagine, grâce au plaisir évident que Constance manifeste en le portant, qu'il retient quelque chose de la chaleur du corps de Parkin et qu'il est doux à l'intérieur, offrant par là une métaphore significative pour définir Parkin, cet homme robuste, un peu fruste d'apparence, mais qui se révèle d'une grande sensibilité. En enfilant le gant de Parkin, on peut aussi penser que, symboliquement, elle apprend à toucher le monde avec le même tact que lui.

13 Cet apprentissage du contact avec la nature semble conditionner et modeler les expériences tactiles plus directement sensuelles et sexuelles qui feront bientôt de ces personnages des amants passionnés. Leurs étreintes seront d'ailleurs souvent liées à la nature : la première fois qu'ils jouissent ensemble, c'est quand ils font l'amour contre un arbre, une autre fois, c'est après avoir couru nus sous la pluie que Parkin prend Constance sur un lit de feuilles humides. Le film met ainsi en scène une sorte de tactilité panthéiste, la caresse sexuelle et le plaisir qu'elle procure étant ici intimement liée à une ouverture tactile au monde.

14 Dans ce film, l'apprentissage du toucher par Constance lui ouvre donc bien des portes, à l'image de la barrière qu'elle doit franchir pour rejoindre la forêt et la cabane de Parkin : il la mène à la rencontre du monde (la nature), de l'autre (Parkin) et d'elle-même (elle découvre le plaisir et réinvente sa vie). La question de l'ouverture est en effet au cœur du film : lors de l'une de leurs rencontres, Parkin invite Constance à le toucher comme pour mieux le découvrir. Le toucher devient ici exploratoire et permet à Constance d'apprivoiser le corps de Parkin, de mieux comprendre ses désirs. Parkin s'ouvre alors totalement à elle ; il se dévoile dans toute sa vulnérabilité, tremblant sous les caresses de Constance. À la fin du film, il dira d'ailleurs que ce qui l'émeut tant dans leur histoire c'est la manière dont ils se sont ouverts l'un à l'autre. Le toucher, ici, unit, il est ouverture à l'autre, échange réciproque.

15 Cela dépasserait le cadre de cet article que de proposer une analyse détaillée d'autres films qui proposent aussi une vision du contact comme rencontre véritable, échange, voire fusion, mais il est intéressant de noter que c'est une expérience similaire du toucher que met par exemple en scène Claire Denis dans Vendredi soir (2002). Le film fait également un éloge du toucher comme plaisir sensuel et sexuel, comme rencontre intime avec l'autre et comme expérience émancipatrice de conventions sociales aliénantes.

16 Souvent liée à la sexualité, l'expérience du toucher peut aussi s'en distinguer : la caresse affectueuse, le massage, la poignée de main amicale entrent dans la catégorie du contactlien et du contact-plaisir qui, sans nécessairement l'exclure, n'est pas directement liée à l'activité sexuelle. Par exemple, dans Nénette et Boni (1996) de Claire Denis, la sensualité du toucher passe par des contacts avec des textures et des matières agréables : avec un plaisir évident, la boulangère plonge ses doigts dans un gâteau crémeux pour récupérer un ticket de tombola ; Boni, lui, caresse avec soin son lapin blanc au pelage soyeux, malaxe des brioches ou pétrit de la pâte à pizza avec volupté. Ces diverses expériences tactiles présentent le toucher sur le mode du plaisir, de l'échange, voire de la connexion intime et épanouissante avec l'autre ou le monde, comme le moyen d'une présence immédiate et continue à l'autre, aux choses et à soi-même. 
17 Toutefois, l'expérience du toucher est plus souvent mise en scène sur le mode du manque et de la frustration dans ce cinéma des sensations. L'expérience tactile est désirée mais refusée, retirée ou impossible, engendrant par conséquent des sentiments disjonctifs de privation, de déception ou de séparation qui s'opposent aux notions traditionnellement attachées à l'idée de toucher (lien, fusion, échange).

Dans Camille Claudel 1915 (2013) de Bruno Dumont, la sculptrice, internée par sa famille dans un asile du Sud de la France, ne peut plus exercer son art, assouvir son désir de façonner la matière pour y imprimer la marque de son intériorité. Dans une scène d'une amertume infinie, elle arrache du sol un bout de terre qu'elle malaxe compulsivement avant de le rejeter avec violence ; la matière, impropre au modelage, résiste au toucher de l'artiste. Loin de son atelier, dans cet asile où tout est minéral et sec, l'expérience du toucher qui définit sa pratique artistique lui est refusée.

Plus souvent, toutefois, c'est entre les êtres que se pose la question du toucher sur le mode du manque ou de la frustration. Dans Romance (1999) de Catherine Breillat, bien qu'il l'aime toujours, Paul n'a plus de désir pour Marie. Il décline tous les rapports charnels qu'elle tente d'initier et refuse même de la toucher ou d'être touché. Cette relation frustrante entraîne Marie à le tromper avec des inconnus et à développer une relation sadomasochiste avec Robert. L'expérience du toucher est donc désolidarisée des sentiments d'amour et de tendresse, ce que confirme une scène à l'humour grinçant où des étudiants en médecine palpent tour à tour l'utérus de Marie lors d'un exercice de travaux pratiques. L'histoire d'amour de Marie et Paul est comme annulée par cette absence de contact comme le suggère l'affiche française du film où le titre, Romance, est barré d'une croix (bien sûr, le X est aussi le signe du cinéma pornographique vers lequel tend le film). La romance, ici, est donc peut-être davantage celle qui s'ébauche entre Marie et Robert.

Pourtant, même dans sa relation avec Robert, l'expérience du toucher est ambiguë puisque sadomasochiste : elle relève à la fois du plaisir et de la douleur. Cette relation place Marie dans une relation de domination : attachée lors de leurs rapports, elle est soumise aux désirs (certes courtois) de Robert. La plus épanouissante des relations qu'elle parvient à établir avec un autre être reste donc une relation de pouvoir, si bien que le contact, ici, ne semble pas autoriser d'échanges réciproques qui conditionnent l'actualisation de la promesse fusionnelle du toucher. Dans ce film, l'expérience tactile est donc soit refusée soit inégalitaire et semble témoigner, en définitive, de l'irréductible séparation des êtres, de leur impossible union, même au cœur des contacts les plus intimes.

21 Dans Chocolat (1988) de Claire Denis, la tension sexuelle entre Aimée, la jeune femme blanche d'un colon installé au Cameroun, et Protée, le domestique noir de la maison, ne peut trouver d'issue épanouissante dans le contact charnel. Lorsqu'un soir Aimée tente d'effleurer la jambe de Protée, celui-ci la repousse brusquement, conscient de l'impossibilité d'une relation (réciproque). Dans ce film, cette impossibilité du toucher rejoue et dramatise la puissante division raciale qui sépare les êtres dans le régime colonial.

Dans Nénette et Boni, le désir charnel que Boni éprouve pour la boulangère de son quartier reste à l'état de fantasme et engendre donc des frustrations bien qu'il trouve des issues temporaires dans un autoérotisme alimenté par des objets fantasmatiques. Lors d'une séquence qui met en scène un rêve à demi conscient de Boni dans lequel il fantasme sur la 
boulangère, le jeune homme associe des images érotiques de cette femme au corps voluptueux avec le son, bien réel lui, de la cafetière qui s'est mise en marche. Le ronron de la cafetière semble alors évoquer, dans son rêve, les manifestations sonores du plaisir de la boulangère. Lorsqu'il se réveille, encore sous l'emprise de son fantasme, il caresse la cafetière qui se trouve au bord de son lit. Ici, le toucher vaut donc pour sa valeur métonymique ou symbolique : le contact désiré avec la femme étant impossible, la cafetière prend une dimension substitutive comme la peluche peut venir compenser l'absence de la mère chez l'enfant.

Dans une autre séquence tout aussi onirique, Boni se réveille un matin et découvre, en sortant de sa chambre, une rangée de brioches jalonnant le sol. Il en ramasse une, la caresse, lui dit bonjour, la croque, en malaxe une autre au son, toujours, de la cafetière. À nouveau ici, le toucher n'a de sens que dans le régime symbolique des fantasmes de Boni : les brioches, associées à la boulangère, fonctionnent comme des objets sexuels fantasmatiques, des fétiches à valeur métonymique, transitionnelle et compensatoire. Cette médiatisation du désir ne fait donc que souligner la séparation avec l'objet réel du désir.

Tout un réseau d'objets se charge ainsi d'une force fantasmatique : par exemple, une image montre le lapin blanc de Boni encadré par les pieds de la boulangère, chaussés d'espadrilles surmontées d'un pompon de fourrure pâle. Cette image-fantasme explique rétrospectivement pourquoi, quelques scènes plus tôt, après avoir uriné en tenant son lapin d'une main, Boni commence à se masturber, la fourrure du lapin étant associée à la femme, à ses souliers, à ses tenues pastelles, aux tissus doux qu'elle porte et peut-être, plus symboliquement, à ses poils pubiens. Ces objets ne sont donc efficaces, et leur contact satisfaisant, que par la force fragile de l'illusion et du désir.

Une scène semble suggérer cette fragilité de l'illusion : celle où Boni, qui est pizzaïolo, pétrit de la pâte à pizza dont la forme ronde rappelle les courbes de la boulangère et qui, ici encore, fonctionne comme objet métonymique. Boni caresse la pâte, la tapote, l'embrasse, l'étire, y enfonce ses doigts dans un mouvement de tension sexuelle évidente. Lorsque cette tension atteint son apogée, il plonge son visage dans la pâte et on ne sait pas bien s'il a joui (bien qu'il ne se soit pas masturbé) ou si, plus vraisemblablement, il est tout simplement frustré car l'illusion, sur laquelle repose l'efficacité fantasmatique de cet objet transitionnel, retombe (comme la pâte) et, avec elle, la possibilité d'en jouir.

Dans ces films, l'expérience du toucher est refusée ou insatisfaisante, elle se vit sur le mode de l'absence et de la séparation ou bien elle est compensatoire et n'a, en définitive, rien d'apaisant ou d'épanouissant. En mettant en scène des personnages qui paraissent incapables d'établir des contacts intimes et réciproques, ces films semblent donc souligner l'étrangéité et la séparation des êtres entre eux.

Enfin, ce cinéma des sensations met souvent en scène l'expérience du toucher dans ses aspects les plus morbides, dans des expériences limites telles le viol, la mutilation, la morsure qui déchire, l'étreinte qui étrangle... Comme dans beaucoup d'autres films, en effet, les contacts y sont souvent solidaires de violences physiques : Sentein donne un coup de poing à Galoup dans Beau Travail (1999) de Denis, le groupe d'amis de la Vie de Jésus (1997) de Dumont tabasse à mort un jeune homme qui a osé sortir avec la copine de l'un d'entre eux, les clients de la prostituée dans la Vie Nouvelle (2002) de Grandrieux la battent et la maltraitent. Cette violence physique cristallise d'ailleurs d'autres violences, celle autoritaire de Galoup dans Beau Travail, la violence sociale qui exclut les jeunes de la 
Vie de Jésus d'une vie active et épanouissante, celle politique et sociale de l'Europe de l'Est dans la Vie Nouvelle.

Le viol est une autre forme de cette violence que peut prendre le toucher quand il devient non plus échange mais appropriation, quand la relation n'est plus réciproque mais unilatérale. La plupart des films de Bruno Dumont posent la question du viol : dans l'Humanité (1999), une fillette est retrouvée morte dans un champ après un viol sordide, dans Twentynine Palms (2003), un homme se fait brutalement violer par un autre homme sous les yeux de sa compagne, Flandres (2006) met en scène le viol collectif d'une femme par un groupe de soldats.

Mais c'est Gaspar Noé qui l'aborde le plus frontalement : dans Irréversible (2002), il met en scène une longue séquence de viol extrêmement brutale, filmée en plan séquence et en plan fixe dans toute sa durée, sans musique. La scène est hyperréaliste et montre le viol dans sa violence la plus abjecte. La pénétration de l'autre, non consentie mais subie, peut être lue ici comme une modalité perverse du toucher qui procure à l'agresseur la jouissance morbide d'éprouver son pouvoir en s'appropriant indûment un autre corps.

Dans le viol, le toucher n'est donc bien sûr plus échange mais soumission, assujettissement. Ces nombreuses représentations du viol soulèvent des questions socioculturelles, notamment dans la lignée des théories féministes, mais aussi existentielles : la brutalité bestiale de ces viols témoigne d'une jouissance sadique que génère non la rencontre mais la profanation, la dégradation et même la destruction de l'autre. La notion de toucher en tant que présence à l'autre et échange réciproque, qui présuppose la reconnaissance de l'autre en tant que tel, est ainsi détruite par le viol qui est un déni pur et simple de l'altérité.

31 De même, le toucher n'est plus contact mais déchirure, destruction dans l'expérience de la mutilation ou de la strangulation. Dans l'Apollonide (2011) de Bonello, un client inflige à une prostituée une mutilation terrible de la bouche, l'affublant d'un sourire funeste. Toujours chez Bonello, dans Tirésia (2003), un homme crève les yeux d'un transsexuel dont il ne supporte pas la métamorphose. Dans Trouble Every Day de Denis, Coré et Shane souffrent d'une terrible pathologie : ils sont animés de violentes pulsions sexuelles qui les poussent à tuer leurs partenaires au cours de l'acte par des pratiques cannibales. Comme si le toucher ne leur permettait pas un contact assez intime avec leur partenaire, ils ressentent le désir de les mordre, de goûter leur sang et leur chair, c'est-à-dire, littéralement, de vampiriser l'autre, de l'incorporer. Similairement, dans Sombre (1998) de Grandrieux, le personnage ne peut jouir avec une femme que s'il l'étrangle.

Dans ces expériences limites qui sont toutefois légion dans ce cinéma des sensations, le toucher, destructeur, devient une expérience morbide qui est souvent solidaire d'un désir chimérique d'appropriation d'autrui. Ce désir de possession et de maîtrise nie voire détruit l'autre. Or la possibilité de rencontre, voire de fusion, qu'offre le toucher ne peut s'actualiser que dans la reconnaissance de l'altérité. Ces films ouvrent donc une béance au cœur de la notion de toucher, traditionnellement comprise comme expérience de la liaison entre les êtres, du contact réciproque, de l'union. 


\section{Le toucher du film et la tactilité des spectateurs : régime haptique et expériences incarnées du cinéma}

33 Le cinéma des sensations fait plus que mettre en scène les expériences tactiles de ses personnages. Outre son intérêt pour les corps et leurs sensations, ce cinéma se caractérise par son esthétique qui joue sur la capacité synesthésique des images et des sons à évoquer d'autres sens et notamment à suggérer des impressions tactiles (de texture, de volume, de densité, de température...). Ce cinéma invite ainsi à adopter un régime haptique de regard et d'écoute qui encourage un mode de perception visuelle et auditive proche du sens du toucher, où l'œil et l'oreille deviennent sensibles à des qualités qui sont d'ordinaire perçues à travers notre contact tactile avec le monde.

Reprenant le terme « haptique » des analyses d'Alois Riegl, Gilles Deleuze a développé une opposition entre espace haptique et espace optique qu'il applique à sa réflexion sur l'art. Il définit l'espace optique ou " strié " comme un espace structuré, hiérarchisé, où la profondeur visuelle permet aux formes de se détacher nettement du fond. Selon Gilles Deleuze, l'espace optique, qu'utilisent notamment les représentations perspectivistes, invite ainsi à une vision éloignée, relativement abstraite et solidaire d'une logique essentialiste. En effet, cette forme de vision distanciée doit identifier l'articulation souvent figée des différents plans, le découpage formel des objets, leurs contours précis, etc. Au contraire, Deleuze définit l'espace haptique ou " lisse » comme un espace de proximité, sans profondeur visuelle, c'est-à-dire comme un espace aformel, sans hiérarchies. L'espace haptique est ainsi un espace d'immédiateté et de contact qui, en favorisant une forme de vision rapprochée, permet au regard de " palper » 1 'objet. $^{5}$

Si Gilles Deleuze mobilise cette distinction conceptuelle pour l'analyse d'œuvres picturales, il est pertinent de l'adapter, comme l'a fait Laura U. Marks, au champ cinématographique ${ }^{6}$. En effet, à travers de (très) gros plans, des mouvements de caméra caressants, une attention particulière aux effets de matière et de surface, un modelage de la lumière ou encore un travail sur la texture des sons, le cinéma peut favoriser un mode tactile de regard et d'écoute, où les yeux et les oreilles, fonctionnant comme des organes du toucher, nous donnent parfois la sensation de pouvoir toucher à notre tour le film.

À l'exception de certains genres comme le film d'horreur, le cinéma « contemplatif » ou certains films d'action, la production cinématographique dominante a tendance à privilégier une vision optique relativement distanciée et intellectualisante en proposant le plus souvent des images et des sons dont la valeur principale est informative : plus que d'éprouver leur impact sensoriel, il s'agit alors pour les spectateurs de repérer, de comprendre et de restituer leur signification dans l'économie de la trame narrative. Au contraire, le cinéma des sensations cherche souvent à encourager une vision haptique dont le fonctionnement, influencé par d'autres facultés sensorielles (tactile, kinesthésique, proprioceptive) et plus proche d'une sensibilité baroque, chaotique, implique davantage la corporéité des spectateurs. ${ }^{7}$

Le cinéma français des sensations mérite donc son appellation à deux titres : d'abord, parce que jamais peut-être le cinéma ne s'était tant intéressé à la représentation et à la mise en scène des sensations, mais aussi parce que ce cinéma crée un régime hyperesthésique du film par la mise en œuvre de tout un éventail de techniques cinématographiques qui permettent d'exploiter les capacités synesthésiques et 
notamment haptiques des images et des sons afin d'offrir aux spectateurs des expériences cinématographiques multisensorielles.

Le (très) gros plan est l'un des outils filmiques les plus efficaces pour inviter les spectateurs à adopter un regard haptique. Claire Denis, notamment, utilise souvent ce type de cadrage pour filmer les corps et les matières. Dans une séquence de Nénette et Boni où Boni fantasme sur la boulangère, un très gros plan révèle un tissu rose à la texture cotonneuse : il faut un moment à l'œil pour comprendre qu'il s'agit de la robe de la boulangère. Ce moment, cet intervalle c'est sans doute celui qu'il faut à l'œil pour changer de mode, pour passer d'un mode optique, distancié, à un mode haptique où l'œil devient réceptif aux stimuli tactiles de l'objet présenté : les plis de la robe, son épaisseur, sa souplesse, les formes du corps qu'elle épouse... De même, lorsque Boni se masturbe, la caméra glisse sur son corps en si gros plan qu'il faut quelques secondes au regard pour comprendre quelle partie du corps est filmée. Ici encore, la connaissance et la mémoire tactile que l'on a des corps et des matières peuvent nous donner la réponse avant même que la caméra, en prenant un peu de distance, ne nous le révèle.

Face à ces plans haptiques où l'objet représenté est difficilement reconnaissable, nos doigts savent, reconnaissent l'objet avant même que notre regard ne le confirme comme le suggère Vivian Sobchack dans son essai au titre révélateur What my fingers knew ${ }^{8}$. Alors que le regard optique tend à garder une distance avec l'objet de la vision, le regard haptique s'en rapproche parfois tant qu'il est difficile de l'identifier. Le processus de reconnaissance visuelle de l'objet étant difficile voire impossible en raison d'une trop grande proximité, les spectateurs doivent alors adopter un regard réceptif à des qualités tactiles, le toucher étant un sens qui ne s'opère pas à distance. Le regard optique propose un régime de vision relativement distancié et hiérarchisé, permettant une distinction claire de l'objet et donc une certaine forme de maîtrise intellectuelle. Quant au regard haptique, il engage notre sens tactile, kinesthésique et proprioceptif. Il propose une forme de vision plus charnelle qui, ne permettant pas toujours d'identifier l'objet, encourage un autre mode de relation avec lui qui est moins de la maîtrise que du rapport sensuel de notre corporéité à sa propre corporéité filmique ${ }^{9}$. Dans ce régime haptique, les images et les sons renoncent parfois à leur pouvoir de représentation claire et distincte des objets pour nous les faire mieux toucher des yeux.

Les mouvements de caméra peuvent aussi effleurer, caresser les corps et encourager par là les spectateurs à adopter un regard tactile. Dans une séquence de l'Apollonide, la tenancière de la maison close présente les prostituées à un nouveau client : un travelling de gauche à droite vient mimer le regard du client qui scrute les filles pour faire son choix. Ce lent mouvement de caméra scanne avec sensualité les corps féminins, s'attarde sur quelques visages comme pour nous laisser le temps de les toucher du regard. Dans Nénette et Boni, les mouvements caressants de caméra sont plus nettement encore une invitation à toucher les corps avec nos yeux : tandis que Boni pense à la boulangère, la caméra caresse, par son plan resserré, par son mouvement lent et doux, le papier peint de la chambre de Boni avant de parcourir en très gros plan le dos du jeune homme avec sensualité si bien qu'elle semble être sa partenaire invisible. Ici, le régime tactile du regard est favorisé par la combinaison du très gros plan, du mouvement de caméra caressant et de la durée du plan qui laisse le temps au regard d'explorer l'image.

41 L'insistance de la caméra sur des effets de matière, de surface ou de texture est aussi une invitation à faire l'expérience d'un film sur un mode tactile comme en témoignent les gros plans sur les gâteaux crémeux dans Nénette et Boni, sur les corps voluptueux et les 
chairs rebondies des prostituées dans l'Apollonide, sur la nuque grasse et transpirante du chef de police dans l'Humanité, sur les corps athlétiques, lisses et imberbes des légionnaires dans Beau Travail.

Le son peut aussi être haptique, texturé. Par exemple, dans l'Apollonide, les effets de matière et de texture (robes, draps du lit, canapés...) sont accentués par des gros plans sonores qui en suggèrent la douceur, la rigidité ou encore l'épaisseur. Dans les premières scènes d'Irréversible, Gaspar Noé utilise des infra-sons suffisamment sensibles pour provoquer un sentiment de malaise, de peur voire de nausée qui nous plongent dans un état émotionnel proche de celui des deux personnages masculins.

Les films qui composent ce cinéma français des sensations offrent donc aux spectateurs une nouvelle expérience - charnelle, viscérale - du cinéma en plaçant le corps et ses sensations au cœur de la diégèse et de la représentation mais aussi au centre de leur projet esthétique. En jouant sur la capacité synesthésique des images et des sons, ce cinéma propose des expériences hyperesthésiques qui invitent les spectateurs à faire l'expérience des films avec tous leurs sens. En mobilisant d'autres expériences sensorielles que la vision et l'audition telles que les fonctions tactiles, kinesthésiques et proprioceptives, les images et les sons haptiques invitent les spectateurs à y répondre de manière incarnée, sensuelle.

Ainsi, une utilisation haptique et une approche synesthésique du medium cinématographique permet de transcender sa nature audiovisuelle, d'évoquer d'autres expériences sensorielles, notamment tactiles, et donc d'offrir aux spectateurs une expérience multisensorielle. Privilégier un regard haptique au cinéma, c'est ainsi actualiser la capacité des films à nous toucher, à nous impliquer sensuellement, viscéralement et non pas seulement intellectuellement.

\section{Discours incarnés et narrations haptiques}

Le régime rationnel de la pensée n'ayant pas le monopole de la production de sens, l'abondance de sensations dans ce cinéma n'est pas purement formelle, creuse ou gratuite, mais signifiante. En effet, la signification n'émerge pas seulement au niveau des signes et des symboles, c'est-à-dire au niveau de la narration, mais aussi dans et par le corps et ses sensations. Dans ces films aux récits et aux dialogues relativement minces, la texture des images et des sons prend ainsi en charge de manière incarnée une large partie de ce qui est en jeu. En effet, le récit se développe sur un mode sensible et non pas discursif : on a pu parler de narration plastique (notamment pour décrire le cinéma de Claire Denis) mais on pourrait préférer le terme de narration tactile ou narration haptique tant les histoires que développe ce cinéma sont incarnées dans les corps des personnages, leurs sensations et leurs contacts et, comme l'écrit M. Beugnet, " intégré[es] dans la texture même des images et des sons $»^{10}$.

Dans ces films, le sens surgit ainsi souvent dans les interstices du visible et du dicible : ce qui ne s'exprime pas (ou ne peut s'exprimer) par le langage ou dans un régime optique de vision, résonne ici dans l'ordre du sensible, dans le corps (des personnages, des spectateurs et dans la matérialité même du film). Le propos se lit moins dans les histoires et les dialogues qu'il ne s'éprouve au contact des corps et des sensations que ces films nous donnent à « toucher » avec nos yeux et nos oreilles. Les corps et leurs sensations ainsi que les images et les sons dépassent donc leur traditionnelle fonction de représentation : ils 
ne sont plus seulement les vecteurs d'une histoire, c'est-à-dire au service d'un discours, mais ils sont un discours en eux-mêmes, un discours incarné. Loin d'être un cinéma formaliste voire sensationnaliste, ce cinéma des sensations peut donc être considéré comme une forme de pensée incarnée (" embodied thoughts $»^{11}$ ) qui, enlaçant intimement le sensuel et le conceptuel, explore la capacité du corps et des sensations, des images et des sons, à créer du sens et, in fine, de nouvelles manières de penser.

\section{NOTES}

1. James Quandt, «Flesh and Blood: Sex and Violence in Recent French Cinema », dans Artforum, février 2004.

2. L'auteur traduit.

3. Martine Beugnet, Cinema and Sensations: French Film and the Art of Transgression, Endinburgh University Press, 2007, quatrième de couverture. L'auteur traduit.

4. Voir les analyses de Linda Williams qui soulignent ce déni théorique dans «Film Bodies: Gender, Genre and Excess », Film Quaterly 44, 1991.

5. Gilles Deleuze et Félix Guattari, Mille plateaux, Paris, Minuit, 1980, pp. 614-622.

6. Voir à ce propos les analyses de Laura U. Marks dans The Skin of the Film, Duke University Press, 2000.

7. Voir les analyses sur l'expérience incarnée du spectateur (" embodied spectatorship ») chez Vivian Sobchack, The Address of the Eye: a Phenomenology of Film Experience, Princeton University Press, 1992.

8. Voir Carnal Thoughts: Embodiment and Moving Image Culture, University of California Press, 2004, chapitre 3.

9. Voir les analyses de Laura U. Marks sur le regard haptique comme antithèse d'un regard optique qui cherche à maîtriser, identifier, contrôler dans The Skin of the Film, op.cit.

10. Martine Beugnet, op. cit. L'auteur traduit.

11. À ce sujet, voir les analyses de Vivian Sobchack dans Carnal Thoughts, op. cit.

\section{RÉSUMÉS}

Depuis les années 1990, la critique a repéré l'émergence d'une nouvelle tendance du cinéma français qui tend à se concentrer sur la mise en scène des corps et la représentation de ses sensations. Ce « cinéma des sensations » accorde une place privilégiée au toucher. La question du contact est souvent au cœur des expériences des personnages et se pose donc d'abord au niveau de la narration et de la mise en scène. Le sens du toucher est également mobilisé dans la matière même des films, car ce cinéma joue sur la capacité synesthésique des images et des sons à évoquer d'autres expériences sensorielles, notamment tactiles. Ce cinéma invite ainsi les spectateurs à adopter un régime haptique de vision et d'écoute, où l'œil et l'oreille deviennent 
sensibles à des qualités tactiles. Ce type de cinéma suscite donc chez les spectateurs une relation incarnée avec le film, induisant une expérience non seulement intellectuelle mais aussi viscérale qui est elle-même porteuse de sens. En effet, le sens de ces films n'émerge pas seulement au niveau de la narration et des dialogues mais aussi dans les sensations qu'ils représentent et suscitent. Il s'agit moins de lire ces films que de les sentir ou de les toucher en prenant en compte la sensualité de leurs représentations et la matérialité même du medium.

Since the 1990s, critics have noticed the emergence of a new tendency in French cinema that tends to focus on representing the body and its sensations. This "cinema of sensations" especially addresses the sense of touch. The question of touch is first raised in the narrative and the miseen-scene as it is often key in the characters' experiences. The sense of touch is also conjured up in the very materiality of the film, as these films play on the synesthetic qualities of the images and sounds to evoke other, particularly tactile, sensory experiences. This invites the spectator to adopt a haptic visuality and hearing, in which the eyes and the ears become sensitive to tactile qualities. Thus, this cinema induces an embodied spectatorship, i.e. an experience that is not only intellectual but also visceral and that is significant by itself. Indeed, in these films, significance does not only emerge at the level of narrative and dialogue but also from the sensations they represent and provoke. To make sense of them, one has less to read them than to feel or touch them by taking into account the carnality of their representations and the sensuality of their materiality.

\section{AUTEUR}

\section{SOPHIE WALON}

Doctorante et chargée d'enseignements en études cinématographiques à l'École Normale Supérieure de Paris sous la direction de Jean-Loup Bourget (ENS Paris) et d'Isabelle Launay (Paris VIII). Ses recherches portent sur le corps et les sensations dans les films de danse expérimentaux. Sophie Walon a écrit de nombreux articles critiques pour la rubrique cinéma du journal Le Monde en 2011 et 2012. Elle est aussi l'auteur de plusieurs articles universitaires : « Figures d'un réalisateur : Chris Marker », Implications philosophiques (revue électronique), juillet 2011 ; « Les corporéités de la danse contemporaine française expérimentale : une pratique philosophique et politique de résistance ", Agôn (revue électronique), novembre 2011 ; « Splendeurs et misères de la chair : corps et sensations dans le cinéma de Bertrand Bonello", Corps : méthodes, discours et représentations (article en ligne), mars 2013 ; « Corporeal Creations in Experimental Screendances: Resisting Socio-political Constructions of the Body » dans The Oxford Handbook of Screendance Studies, Oxford University Press, (date de publication prévue : début 2014). 